\title{
A COMPACT REGULAR OPERATOR WITHOUT MODULUS
}

\author{
Y. A. ABRAMOVICH AND A. W. WICKSTEAD \\ (Communicated by Palle E. T. Jorgensen)
}

\begin{abstract}
We construct an operator with the properties mentioned in the title. Without the regularity assumption such an example was first discovered by $U$. Krengel.
\end{abstract}

\section{INTRODUCTION}

It is a classical result due to Krengel [K1] (see also [AB, Example 16.6]) that a compact operator on a Banach lattice does not necessarily have a modulus. The aim of this article is to strengthen this result by showing that, even under the additional assumption of regularity, a compact operator may still not have a modulus. Recall that an operator is regular if it can be written as the difference of two positive operators. It follows from our example that there are compact sets in Banach lattices that are bounded above but fail to possess a supremum (else we could use the Riesz-Kantorovich formula to construct the modulus of a compact regular operator). Since this rather fundamental counterexample seems to have been overlooked in the literature, we present in the next section a direct construction of such a set. This result should be compared with another classical result of Krengel [K2] that each compact subset $A$ of an arbitrary $C(K)$-space has a supremum, and consequently, each compact operator $T: X \rightarrow C(K)$, where $X$ is any Banach lattice, has a modulus. Some relevant information about the characterizations of compact subsets of AM-spaces may be found in $[\mathrm{AB}, \mathrm{Ab} 1, \mathrm{~W}]$.

We refer the reader to $[\mathrm{AB}, \mathrm{S}, \mathrm{V}, \mathrm{Z}]$ for the basic definitions in this topic.

\section{A COMPACT SET THAT IS BOUNDED ABOVE BUT HAS NO SUPREMUM}

Before presenting the example, let us establish some notation. If $E$ is a Banach lattice, we shall denote by $c(E)$ the space of all norm convergent sequences $\left(x_{n}\right)$ from $E$; when given the usual linear operations, the coordinatewise order and the norm $\left\|\left(x_{n}\right)\right\|=\sup \left\{\left\|x_{n}\right\|_{E}: n \in \mathbb{N}\right\}$, this becomes a Banach lattice. If $\left(E_{n}\right)$ is a sequence of Banach lattices, then the space $c_{0}\left(E_{n}\right)$ of all sequences

Received by the editors November 12, 1990 and, in revised form, April 1, 1991.

1991 Mathematics Subject Classification. Primary 47B65, 47B06; Secondary 46B42, 46A50.

Key words and phrases. Banach lattice, compact set, compact regular operator.

This work was made possible by a NATO Collaborative Research Grant CRG-890909 that allowed Dr. Wickstead to visit IUPUI during the Summer of 1990. 
$\left(x_{n}\right)$ such that $x_{n} \in E_{n}$ and $\left\|x_{n}\right\| \rightarrow 0$, is also a Banach lattice under the same operations and norm. We will denote by 1 the constantly one sequence in $c$, the space of all convergent real-valued sequences, and by $e_{n}$ the real sequence with all terms zero except for the $n$ th, which is one. Similarly we will use the notation $\mathbf{1}_{[0,1]}$ to denote the constantly one function on $[0,1]$. If $\left(x_{n}\right) \in c$ and $\varphi \in E$, then $\left(x_{n}\right) \otimes \varphi$ will denote that element of $c(E)$ with $n$th term $x_{n} \times \varphi$, the usual product of the element $\varphi \in E$ by the scalar $x_{n}$.

Define a set of elements $A=\left\{y^{n, k}: 1 \leq k \leq n, n \in \mathbb{N}\right\} \cup\{0\}$ in $c\left(L^{1}[0,1]\right)$ as follows. Let $y^{n, k}$ be the sequence with all entries zero except for the $2 n$th one, which is the characteristic function of the interval $[(k-1) / n, k / n]$. Then we see that $\left\|y^{n, k}\right\|=1 / n$ so that the set $A$ is certainly norm-compact. The sequence $\mathbf{1} \otimes \mathbf{1}_{[0,1]}$ is in $c\left(L^{1}[0,1]\right)$ and is an upper bound for $A$. It remains only to show that the set $A$ has no supremum.

If some $\left(z_{n}\right) \in c\left(L^{1}[0,1]\right)$ is any upper bound for $A$, then we have $z_{2 n} \geq 1$ on each interval $[(k-1) / n, k / n]$ and, therefore, $z_{2 n} \geq 1$ on the whole of $[0,1]$. Clearly also $z_{2 n+1} \geq 0$. Conversely if $z_{2 n} \geq 1$ on $[0,1]$ and $z_{2 n+1} \geq 0$, then $\left(z_{n}\right)$ will be an upper bound for $A$. We show now that such an upper bound $\left(z_{n}\right)$ cannot be a supremum. Since $z_{n} \rightarrow z_{\infty} \in L^{1}[0,1]$, we see that $z_{\infty}=\lim z_{2 n} \geq 1$ on the whole of $[0,1]$. Choose $n_{0}$ such that $n \geq n_{0}$ implies that $\left\|z_{n}-z_{\infty}\right\|_{1}<\frac{1}{2}$; then for such $n$ we have $\left\|z_{n}\right\|_{1} \geq\left\|z_{\infty}\right\|_{1}-\frac{1}{2} \geq \frac{1}{2}$. In particular, if $n>n_{0}$ then $z_{n} \neq 0$. But if $\left(z_{n}\right)$ is an upper bound for $A$, then picking any odd integer $m>n_{0}$ and replacing $z_{m}$ by the zero function in $L^{1}[0,1]$ gives us an upper bound for $A$ that is strictly less than $\left(z_{n}\right)$. It follows that $A$ has no supremum.

Note that a Banach lattice containing a set like $A$ cannot be Dedekind complete. Otherwise, being order bounded, $A$ would have a supremum.

\section{A REGULAR COMPACT OPERATOR WITH NO MODULUS}

Before presenting this example let us recall from [AW] the following result, which is easily proved by induction.

Lemma. For any elements $x_{1}, x_{2}, \ldots, x_{n}$ in a Riesz space

$$
\sup \left\{\sum_{k=1}^{n} \varepsilon_{k} x_{k}: \varepsilon_{k} \in\{-1,0,1\}\right\}=\sum_{k=1}^{n}\left|x_{k}\right| .
$$

Let $l_{m}^{\infty}$ denote $m$-dimensional real space with the pointwise order and the supremum norm $\|\cdot\|_{\infty}$. It is routine to verify that $c_{0}\left(l_{2^{n}}^{\infty}\right)$ is norm and order isomorphic to the familiar sequence space $c_{0}$. Let $r_{n}$ denote the $n$th Rademacher function on $[0,1]$ (see [AB, p. 161]). The only properties that we need of these functions are that they are orthonormal in $L^{2}[0,1]$ and all have modulus that is constantly one (almost everywhere). Our example will be an operator from the sequence space $c$ into $c\left(L^{2}[0,1]\right)$.

Define an operator $T_{n}: l_{2^{n}}^{\infty} \rightarrow c\left(L^{2}[0,1]\right)$ by

$$
T_{n}\left(\alpha_{k}\right)= \begin{cases}\frac{1}{2^{n}} e_{n} \otimes \sum_{k=1}^{2 n} \alpha_{k} r_{k} & (n \text { odd }), \\ 0 & (n \text { even }) .\end{cases}
$$

Using the orthogonality of the Rademacher functions we see that if $n$ is odd 
then

$$
\begin{aligned}
\left\|T_{n}\left(\alpha_{k}\right)\right\| & \leq \frac{1}{2^{n}}\left\|e_{n}\right\| \times\left\|\sum_{k=1}^{2^{n}} \alpha_{k} r_{k}\right\|=\frac{1}{2^{n}} \sqrt{\sum_{k=1}^{2^{n}}\left|\alpha_{k}\right|^{2}} \\
& \leq 2^{-n / 2}\left\|\left(\alpha_{k}\right)\right\|_{\infty},
\end{aligned}
$$

so that $\left\|T_{n}\right\| \leq 2^{-n / 2}$ no matter whether $n$ is odd or even. It follows that we may define a compact operator on $c_{0}\left(l_{2^{n}}^{\infty}\right)$ by $T\left(x_{n}\right)=\sum T_{n} x_{n}$. If we define $T \mathbf{1}=0$ and use the identification of $c_{0}\left(l_{2^{n}}^{\infty}\right)$ with $c_{0}$, then we have produced a compact operator from $c$ into $c\left(L^{2}[0,1]\right)$.

Define also $S_{n}: l_{2^{n}}^{\infty} \rightarrow c\left(L^{2}[0,1]\right)$ by

$$
S_{n}\left(\alpha_{k}\right)=\frac{1}{2^{n}}\left(\sum_{k=1}^{2^{n}} \alpha_{k}\right) e_{n} \otimes \mathbf{1}_{[0,1]}
$$

for all $n \in \mathbb{N}$. Note that $\left\|S_{n}\right\|=1$. Observe now that if $F$ is a finite subset of $\mathbb{N}, x_{n} \in l_{2^{n}}^{\infty}$ and $\left\|x_{n}\right\|_{\infty} \leq \varepsilon$ for all $n \in F$, then $\left\|\sum_{n \in F} S_{n} x_{n}\right\| \leq \varepsilon$ as

$$
\begin{aligned}
\left\|\sum_{n \in F} S_{n} x_{n}\right\| & =\sup _{k \in \mathbb{N}}\left\|\left(\sum_{n \in F} S_{n} x_{n}\right)_{k}\right\|_{2} \\
& =\sup _{k \in \mathbb{N}} \begin{cases}\left\|S_{k} x_{k}\right\|_{2} & \text { if } k \in F, \leq \varepsilon . \\
0 & \text { if } k \notin F\end{cases}
\end{aligned}
$$

It follows that if $x_{n} \in l_{2^{n}}^{\infty}$ and $\left\|x_{n}\right\|_{\infty} \rightarrow 0$, then $\sum S_{n} x_{n}$ converges in norm in $c\left(L^{2}[0,1]\right)$ to an element, which we will denote by $S\left(x_{n}\right)$. Define also $S \mathbf{1}=(2 \times \mathbf{1}) \otimes \mathbf{1}_{[0,1]}$. Obviously $S$ is a linear operator from $c$ to $c\left(L^{2}[0,1]\right)$. We claim that $S$ is a positive majorant for $T$.

If $\left(x_{n}\right) \in c_{0}\left(l_{2^{n}}^{\infty}\right)_{+}$, then $x_{n}=\left(\alpha_{1}^{(n)}, \alpha_{2}^{(n)}, \ldots, \alpha_{2^{n}}^{(n)}\right)$, where each $\alpha_{k}^{(n)} \geq 0$, and hence

$$
\begin{aligned}
(S-T)\left(x_{n}\right) & =\sum\left(S_{n}-T_{n}\right) x_{n} \\
& =\sum_{n=1}^{\infty} \frac{1}{2^{n}} e_{n} \otimes\left[\sum_{k=1}^{2^{n}} \alpha_{k}^{(n)}\left(\mathbf{1}_{[0,1]}-r_{k}\right)\right] \geq 0
\end{aligned}
$$

(as $\mathbf{1}_{[0,1]} \geq r_{k} \geq-\mathbf{1}_{[0,1]}$ ). Also note that

$$
S\left(x_{n}\right)=\sum_{n=1}^{\infty} S_{n} x_{n}=\sum_{n=1}^{\infty} \frac{1}{2^{n}} e_{n} \otimes\left(\sum_{k=1}^{2^{n}} \alpha_{k}^{(n)}\right) \geq 0 .
$$

In order to complete a proof that $S \geq T, 0$ we need to consider positive elements of $c$ of the form $\mathbf{1}+\left(x_{n}\right)$, where $\left(x_{n}\right) \in c_{0}\left(l_{2^{n}}^{\infty}\right)$. By the positivity of the sum, $x_{n} \geq(-1,-1, \ldots,-1)$, where there are exactly $2^{n}$ terms equal to -1 . Thus

$$
\begin{aligned}
S\left(\mathbf{1}+\left(x_{n}\right)\right) & \geq(2 \times \mathbf{1}) \otimes \mathbf{1}_{[0,1]}+\sum_{n=1}^{\infty} \frac{1}{2^{n}} \sum_{k=1}^{2^{n}}(-1) e_{n} \otimes \mathbf{1}_{[0,1]} \\
& =(2 \times \mathbf{1}) \otimes \mathbf{1}_{[0,1]}+\sum_{n=1}^{\infty}\left(-e_{n}\right) \otimes \mathbf{1}_{[0,1]} \geq 0
\end{aligned}
$$


and

$$
\begin{aligned}
(S-T)\left(\mathbf{1}+\left(x_{n}\right)\right) & \geq(2 \times \mathbf{1}) \otimes \mathbf{1}_{[0,1]}+\sum_{n=1}^{\infty} \frac{1}{2^{n}} e_{n} \otimes \sum_{k=1}^{2^{n}}-\left(\left|r_{k}\right|+1\right) \\
& =(2 \times \mathbf{1}) \otimes \mathbf{1}_{[0,1]}-2 \sum_{n=1}^{\infty} e_{n} \otimes \mathbf{1}_{[0,1]} \geq 0 .
\end{aligned}
$$

It follows that $S \geq T, 0$ and, therefore, $T=S-(S-T)$ is a regular operator.

It remains to prove that $T$ fails to have a modulus. If $U \geq T,-T$ then on each $l_{2^{n}}^{\infty}$, for $n$ odd, we have

$$
(1,1, \ldots, 1) \geq\left(\varepsilon_{1}, \varepsilon_{2}, \ldots, \varepsilon_{2^{n}}\right) \geq(-1,-1, \ldots,-1)
$$

for any choice of $\varepsilon_{k} \in\{-1,0,1\}$. Thus

$$
U(1,1, \ldots, 1) \geq T_{n}\left(\varepsilon_{1}, \varepsilon_{2}, \ldots\right)=\frac{1}{2^{n}} e_{n} \otimes \sum_{k=1}^{2^{n}} \varepsilon_{k} r_{k}
$$

and by the lemma we have

$$
U(1,1, \ldots, 1) \geq \frac{1}{2^{n}} e_{n} \otimes \sum_{k=1}^{2^{n}}\left|r_{k}\right|=e_{n} \otimes \mathbf{1}_{[0,1]} .
$$

This happens for all odd $n$, so certainly $\lim _{n \rightarrow \infty}(U \mathbf{1})_{n} \geq \mathbf{1}_{[0,1]}$. Since each $T x$ vanishes on even coordinates in $c\left(L^{2}[0,1]\right)$, we see that if $|T|$ existed it would have $(|T| x)_{2 n}=0$ for all $n$ and $x$. In particular, $(|T| \mathbf{1})_{2 n}=0$ and hence $\lim _{n \rightarrow \infty}(|T| \mathbf{1})_{n}=0$. As this contradicts the fact that this limit must be at least $\mathbf{1}_{[0,1]}$, this shows that $|T|$ cannot exist.

Notice that, as in the previous section, such an example is only possible if the range space $Y$ is not Dedekind complete. For if $Y$ were Dedekind complete, then by the classical Riesz-Kantorovich theorem, the space $L^{r}(X, Y)$ of all regular operators from any vector lattice $X$ into $Y$ would be a vector lattice (even a Dedekind complete vector lattice) and $|T|$ would exist.

\section{Comments}

(1) The operator $S$ constructed in $\S 3$ is easily seen to be noncompact. It is therefore still possible that every compact regular operator majorized by a compact positive operator has a modulus (and even possible that the modulus must be compact).

(2) In spite of the "negative" nature of the results presented in this article, we still hope that it is possible to impose extra assumptions on a compact operator $T$ that would imply some nontrivial information about $|T|$. For example, the assumption in (1) might suffice.

On the other hand, we would like to stress that such additional hypothetical conditions will have to be applied to an individual operator rather than to the whole class of compact operators. Otherwise, as the following result (see [Ab1, Corollary to Theorem 5.2]) says, the nature of the space $Y$ is predetermined.

Let $X$ and $Y$ be Banach lattices, where $X$ is not a KB-space, i.e., $X$ contains a subspace isomorphic to $c_{0}$. Then the following four conditions are equivalent. (a) $Y$ is an AM-space, (b) for each compact $T: X \rightarrow Y$ there exists $|T|: X \rightarrow Y$, 
which is also compact, (c) each compact operator $T: X \rightarrow Y$ is regular, (d) each compact operator $T: X \rightarrow Y$ is regular as an operator from $X$ to $Y^{* *}$.

Each of the implications (b) $\Rightarrow(a),(c) \Rightarrow(a)$, or (d) $\Rightarrow$ (a) shows that a global condition on the whole space $K(X, Y)$ of compact operators implies immediately that $Y$ is a classical $A M$-space.

(3) Using Khinchin's inequality [LT, Theorem 2.b.3], our example in $\S 3$ may be modified to give an example of a regular compact operator taking values in $c\left(L^{p}[0,1]\right)$, with no modulus, for each $p \in[1, \infty)$. Also we can replace the discrete space $c$ by the space $C[0,1]$ and consider a corresponding analogue of the space $c\left(L^{p}[0,1]\right)$.

(4) The following question was asked in [Ab2] (Question 3, p. 139). Does there exist a pair of Dedekind complete Banach lattices $X$ and $Y$ such that $K(X, Y) \subset L^{r}(X, Y)$ but $L^{r}(X, Y) \neq L(X, Y)$ ? (Here $L(X, Y)$ denotes, as usual, the space of all continuous operators from $X$ to $Y$.)

We give below a positive solution to this problem. Let $X=C(Q)$, where $Q$ is an arbitrary (infinite) extremally disconnected compact space. Let $q_{0}$ be a fixed nonisolated point in $Q$ and let $Y=\left\{f \in X: f\left(q_{0}\right)=0\right\}$. Both $X$ and $Y$ are Dedekind complete AM-spaces. By the theorem cited in (2) $K(X, Y) \subset L^{r}(X, Y)$. Moreover, $|T| \in K(X, Y)$ for each $T \in K(X, Y)$.

We claim that $L(X, Y) \neq L^{r}(X, Y)$. Let $T \in L(X, Y)$ be defined as $(T f)(q)=f-f\left(q_{0}\right) \mathbf{1}$. Obviously $T \in L(X, Y)$ and $T f=f$ for all $f \in Y$. It is easy to see that $T$ is not regular. Indeed, since $Y$ is Dedekind complete, and if $T$ were regular, then there would exist its positive part $T^{+}$, and for each $x \in X_{+}$the value $T^{+} x \in Y$ would be computed by the Riesz-Kantorovich formula. In particular, we would have

$$
T^{+} \mathbf{1}=Y-\sup \{T f: 0 \leq f \leq \mathbf{1}\} .
$$

Observe now that $Y$ is an ideal in $X$ and, therefore, $Y-\sup (D)=X-$ $\sup (D)$ for each order bounded subset $D$ of $Y$.

On the other hand, $Y-\sup \{T f: 0 \leq f \leq \mathbf{1}\}$ cannot exist, since otherwise

$$
\begin{aligned}
T^{+} \mathbf{1} & =Y-\sup \{T f: 0 \leq f \leq \mathbf{1}\} \\
& =X-\sup \{T f: 0 \leq f \leq \mathbf{1}\} \\
& \geq X-\sup \left\{T f: 0 \leq f \leq \mathbf{1} \& f\left(q_{0}\right)=0\right\}=\mathbf{1} .
\end{aligned}
$$

Since $T^{+} \mathbf{1}$ is in the ideal $Y$, this would imply that $\mathbf{1} \in Y$, which is a contradiction.

However, under the natural additional assumption that at least one of the Banach lattices $X$ or $Y$ be reflexive, Question 3 from [Ab2] remains open.

(5) The first author wants to use this opportunity to improve the phraseology of Theorem 8.12 in [Ab2]. The concluding sentence, "Then $S$ is finite," should have read, "Then $C(S)$ is Dedekind complete." The original version is correct but the modification makes it easier to understand the comment that follows Theorem 8.12.

\section{ACKNOWLEDGMENT}

The authors would like to thank the referee for his suggestions. 


\section{REFERENCES}

[AB] C. D. Aliprantis and O. Burkinshaw, Positive operators, Academic Press, New York and London, 1985.

[Ab1] Y. A. Abramovich, Some new characterizations of AM-spaces, Ann. Univ. Craiova 6 (1978), $15-26$.

[Ab2] _ When each continuous operator is regular, Functional Analysis, Optimization and Math. Economics, Oxford Univ. Press, New York and London, 1990, pp. 133-140.

[AW] Y. A. Abramovich and A. W. Wickstead, Regular operators from and into a small Riesz space, Indag. Math. 2 (1991), 257-274.

[Kg1] U. Krengel, Remark on the modulus of compact operators, Bull. Amer. Math. Soc. 72 (1966), 132-133.

[Kg2] _ U Über den Absolutbetrag stetiger linearer Operatoren und seine Anwendung auf ergodische Zerlegung, Math. Scand. 13 (1963), 151-187.

[LT] J. Lindenstrauss and L. Tzafriri, Classical Banach spaces. I, Springer, Berlin, Heidelberg, and New York, 1977.

[S] H. H. Schaefer, Banach lattices and positive operators, Springer, Berlin, Heidelberg, and New York, 1974.

[V] B. Z. Vulikh, Introduction to the theory of partially ordered spaces, Noordhoff, Groningen, 1967.

[W] A. W. Wickstead, Compact subsets of partially ordered Banach spaces, Math. Ann. 212 (1974), 271-284.

[Z] A. C. Zaanen, Riesz spaces. II, North-Holland, Amsterdam, 1983.

Department of Mathematics, Indiana University-Perdue University at INDIANAPOLIS, INDIANAPOLIS, INDIANA 46205

E-mail address: IMQB100@INDYVAX.Bitnet or IMQB100@INDYVAX.IUPUI.EDU

Department of Pure Mathematics, The Queen's University of Belfast, Belfast BT7 INN, NORTHERN IRELAND

E-mail address: A. Wickstead@QUB.AC.UK 